\title{
Empowering Self-Management through M-Health Applications
}

\author{
Muhaini Othman ${ }^{1, *}$, Norhafizah Mohd Hali1 ${ }^{1, * *}$, M. Mohd Yusof $^{1}$, R. Mohamed ${ }^{1}$, and Mohd Hafizul Afifi Abdullah ${ }^{1}$ \\ ${ }^{1}$ Faculty of Computer Science and Information Technology, Universiti Tun Hussein Onn Malaysia, 86400 Parit Raja, Batu Pahat, \\ Johor, Malaysia
}

\begin{abstract}
The advancement in mobile technology has led towards a new frontier of medical intervention that never been thought possible before. Through the development of MedsBox Reminder (MBR) application for Android as a pilot project of M-Health, health care information system for patient selfmanagement is made possible. The application acts as an assistant to remind users for their timely medicine intake by notifying them through their mobile phone. MedsBox Reminder application aims to facilitate in the self-management of patient's health where they can monitor and schedule their own medicine intake more efficiently. Development of the application is performed using Android Studio 1.4, Android SDK, MySQL database, SQLite, Java language and Netbeans IDE 8.1. Object-Oriented System Development (OOSD) methodology has been adapted to facilitate the development of the application.
\end{abstract}

\section{Introduction}

Non-communicable disease such as diabetic, cardiovascular diseases, stroke, and hypertension continues to increase in the communities worldwide [1]. Medical intervention and self-management are critical to ensure the well-being of patients suffer from the noncommunicable disease since they need extra attention for a longer period of time. Family and close relatives could also act as a mediator for patients in monitoring their health. Most patients are elderly people, who tend to ignore their schedule medicine intake and could result in a severe health relapse.

Currently, there is abundance of advertisement of health awareness and mobile applications has been developed that improve the living condition, nevertheless, without a proper health management system to monitor and intervene patients the quality of life is decreased [2]. Toward empowering people in selfmanagement of their health and manage their long-term illnesses conditions systematically, patients must have access to a timely information, advice, assessment, and treatment from the medical practitioners [3]. Thus, the progressive growth of mobile technology, social networking and medical applications made selfmanagement of health and awareness possible.

As a pilot study, a prototype Android application called MedsBox Reminder (MBR) is developed to empower self-management of health, acting as a mobileenhanced preventive care, wellness management system to monitor patient's health and their medicine intake. The application is capable of encouraging self-health management and connects medical practitioners with patients on a personal level for guidance, monitoring and medical intervention.

\section{Related Works}

In this section, the essential background knowledge of mobile health applications and its related works which represents the essence of our work are introduced.

M-Health can be defined as "mobile computing, medical sensor, and communications technologies for health-care" [4]. WHO [5] has similarly emphasizes the application of devices such as mobile phones, patient monitoring devices, personal digital assistants, and other wireless devices for supporting medical and public health practice.

Health care industry has benefited significantly through the advancement of M-Health technology. People are going mobile where $52 \%$ of smartphones users gather health-related information on their phones while $80 \%$ of physicians use smartphones and medical applications [6]. The success of M-Health due to its usability and cost saving, especially in-home health care of the elderly where it increases patient's mobility and accessibility to a better wellness program anytime and anywhere [7]. To enrich the future of M-Health programs, M-Health systems should be inter-operable with the existing e-health standards [8]. Through proper execution, M-Health system may assist in providing compliance patient treatment, health data collection and disease monitoring, health information systems, support tools for medical practitioners, promotes individual health care, disease prevention, emergency medical response systems, economical treatments, appointment reminders, referral of patients, remote health services, super specialist services, emergency medicine, mobile medical data, mobile robotic system, post-hospital care,

* Corresponding author: muhaini@uthm.edu.my 
telecommunication collaboration, medical information, pre-operative, and post-operative treatments [9].

Medical practitioners play an important role in empowering self-health management by giving guidance, monitoring adverse events, and identify areas for improvement while giving patients independently self-management of their health [2]. Through computermediated technologies, systematic intervention from medical practitioners and community is feasible, thus improving quality of life for individuals [10]. A research was carried out to measure user acceptance of M-Health remote monitoring system using a racially diverse sample of stroke patients to enhance medication adherence and blood pressure control [11]. The research has received positive feedback where $85 \%$ of the patients were comfortable with a nurse or doctor monitoring their health information using M-Health systems, while 78.3\% of them believed it could assist medical adherence. $83.3 \%$ were confident this technology is an effective way for them to communicate with their respective health providers [11].

Aside from intervention from the medical practitioners, community or group intervention is also a good practice to promote a healthy lifestyle. There a mobile-based application called NutriWalking (NW), which recommends personalised daily exercise goals and promotes healthy nutritional habits among small groups specifically for patients with type-2 diabetes mellitus and depression. The application gathers peer support from people who suffer from the same disease, and the group members can assist each other through this application, acting as a self-management support tool [12]. Much more application like NW is being developed and can be downloaded via Google Play Store for Android phone users. Some of the M-Health system are for monitoring patient's health or self-monitoring [13-15], reminder [16], diagnosing diseases [17], non-communicable disease prevention [18], and clinical decision making [19].

One research has proposed a low-cost mobile health care monitoring system for monitoring ECG, blood pressure, $\mathrm{SaO}_{2}$ level, and body temperature to facilitate treatments of chronic diseases [13]. The collected data is visualised in Android devices to assist patients in monitoring their health. Nguyen et al. [14] have identified few mobile applications to enhance selfmanagement of patients with gout. These applications allow the users to monitor the symptoms of their gout disease including serum uric acid level, acute attacks, and educate patients about gout. The other application is for self-management and education of cardiac patients fighting against chronic diseases, especially heart diseases, whereby medical and users' activities are recorded including medication time reminder [15]. There are also applications developed to assist in monitoring and prevention of non-communicable diseases such as Stroke Riskometer which provides estimates of the absolute risk of stroke within the next 5 to 10 years for individuals aged 20 years and above, providing user a baseline risk comparison which allows them to compare their stroke risk with someone of the same age and gender who has no risk factors [18]. Since 5 to 10 years prediction of stroke risk is still inadequate, there are several other researches related to personalized modelling using spiking neural networks methods, aimed to predict stroke risk event several days earlier before stroke occurrences [20-21].

Besides monitoring and self-management applications, few works have studied the use of medical applications as a reminder for immunization [16]. Through a survey, the usage of Android applications for immunization reminder has received positive feedbacks from physicians, parents, and guardians. The advancement in mobile technology and authentication leads towards improved home health care where a person who suffers from chronic disease requires a reminder application to signal them in taking medicine promptly [22]. Therefore, Internet of Things (IoT) has become an essential part of data transaction between source points to destination for the reminder applications. Finally, a mobile phone reminder application using text messages was developed for an anti-psychotic patient [23]. This tool is a practical and ideal approach towards aiding different type of mental illness whereby patients can select preferred text messages as a reminder for medication intake, treatment appointments, and support.

In conclusion, monitoring and reminder applications have been helpful for users in assisting them in monitoring and keep track of the patient's health care records as well as their medication intake. This motivates the development of MedsBox Reminder application with the intention to help the medical practitioners to provide patients with the access to the prescribed medication and acts as a reminder for the patients' daily medication intake.

\section{Methodology}

The methodology used for the development of MedsBox Reminder application is Object-Oriented System Development (OOSD) which consists of four phases: i) object-oriented requirement analysis, ii) object-oriented design, iii) object-oriented implementation, and iv) object-oriented testing.

During object-oriented requirement analysis phase, data collection techniques such as observations and questionnaire were utilized to study the pattern of medicine intake among various community groups involving the elderly and youth. Survey and questionnaires were distributed among patients and clinicians, interviews were conducted with registered clinicians and physicians, and observation in clinics and pharmacies were also done. Information gathered includes medicine intake rules and the preferred method to keep in touch with the patients. The respondents were invited to participate in the evaluation of the prototype.

In the object-oriented design phase, information for development of the proposed application is translated into a working prototype. The purpose of the phase is to ensure the developed application meets the end-user's requirements before the prototype is translated to the production application. Unified Modelling Languages (UML) are used as a method of designing activities 
involved in MedsBox Reminder which consists of use cases, sequence diagrams and class activity diagrams.

During the object-oriented implementation phase, the user interfaces for each module in the prototype will be converted into program codes and connected to the database to fulfill specified functionality for the final product. Figure 1 visualises the system architecture where the main entities described as meducal practitioners (clinicians/pharmacists) and patients, will have direct access to the system. Clinicians/pharmacists access the system via administrator website while patients need to download the MedsBox Reminder application and install it on their mobile phone or tablet, to gain access to their record.

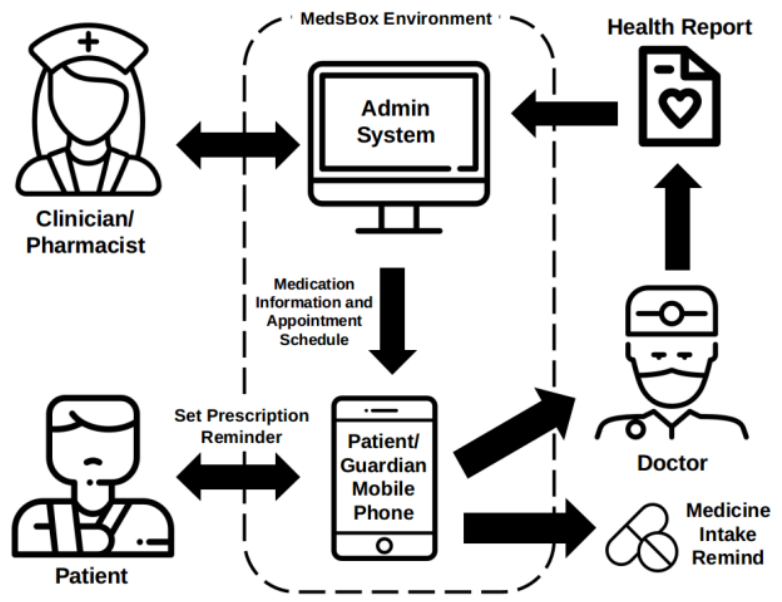

Fig. 1. MedsBox Reminder system architecture.

\section{User Interface and Functionality}

MedsBox Reminder application consists of 5 modules: login module, member registration module, patient's medicine module, appointment module, and medicine module. Patients have access to 3 modules: login module, appointment module, and medicine intake module (with notification reminder).

The administrator modules which are accessible by medical practitioners allows insert/update patient's data such as personal data (Figure 2), medication information for patients (Figure 3), and update a scheduled appointment for patient's check-up (Figure 4).

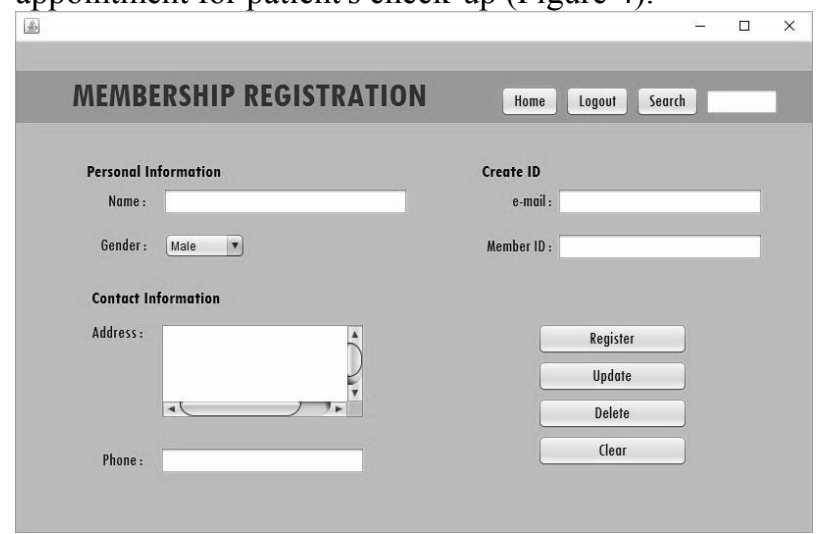

Fig. 2. Patient registration module (Admin).

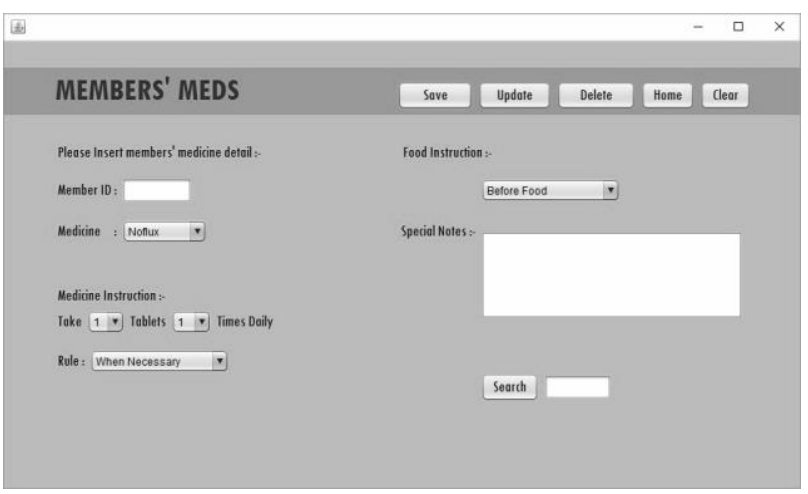

Fig. 3. Patient medicine record module (Admin).

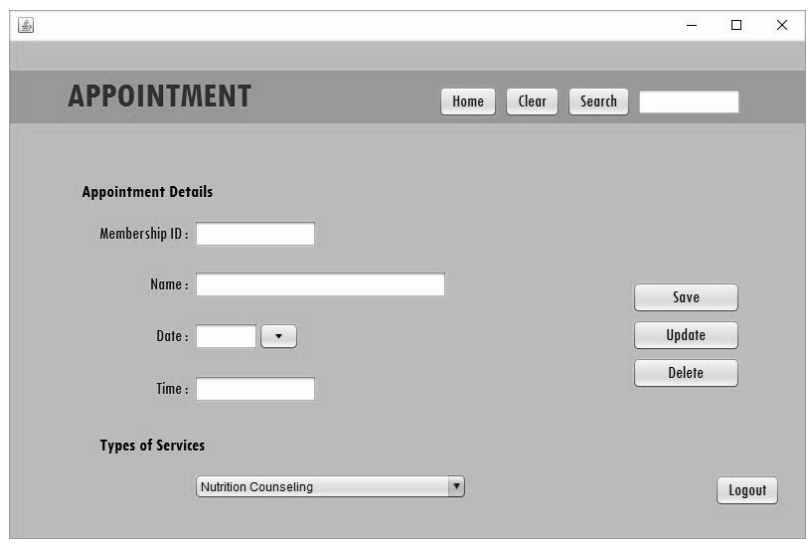

Fig. 4. Patient appointment module (Admin).

The administrator also can insert and update new medicine record into the database through medicine record page (Figure 5). The information about the medicine prescription can be accessed by patients via the MedsBox Reminder application.

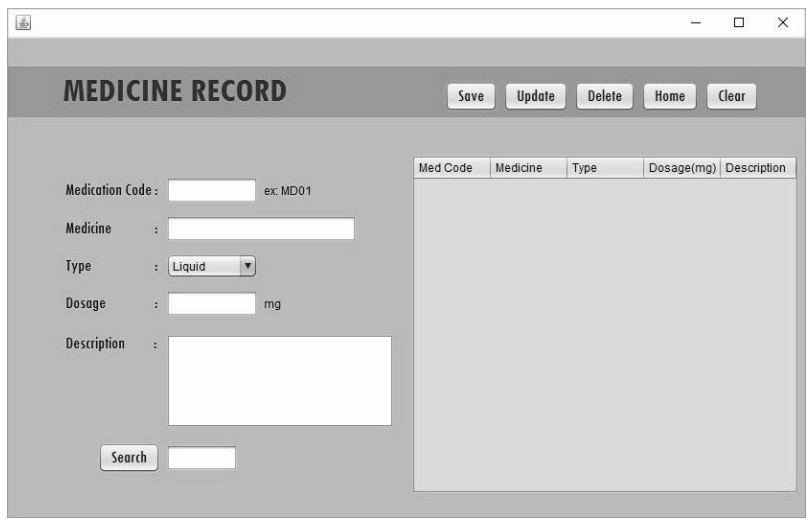

Fig. 5. Medicine record page (Admin).

MedsBox Reminder applications allow patients access to all information regarding medication prescribed for them. The application also provides medicine prescription reminder functionality to schedule personal medicinal intake according to the physicians' direction. Patients are able to view and reschedule their medical appointment via the MedsBox Reminder application. The patients have to $\log$ in into the application to verify that they are registered to the clinic 
or pharmacy. After logging into the mobile application, a menu as in Figure 6 will be displayed.

Figure 7 (a) shows list of medicine intake reminders set, where patients can delete and update previous reminders. The medicine reminder setting page is depicted in Figure 7 (b). Through this module, the user can schedule their medicine intake according to the instruction from clinicians/pharmacists.

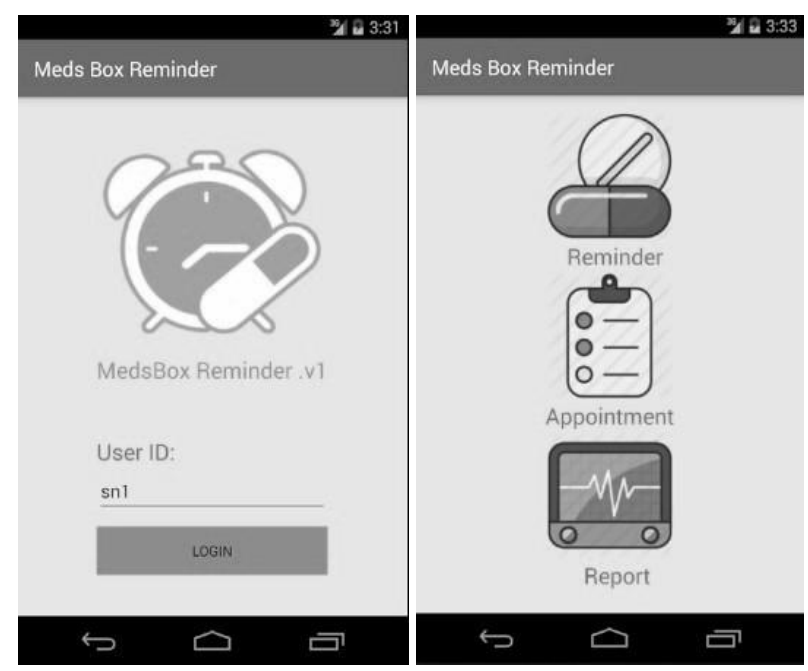

Fig. 6. Login module and the menu (Patient).

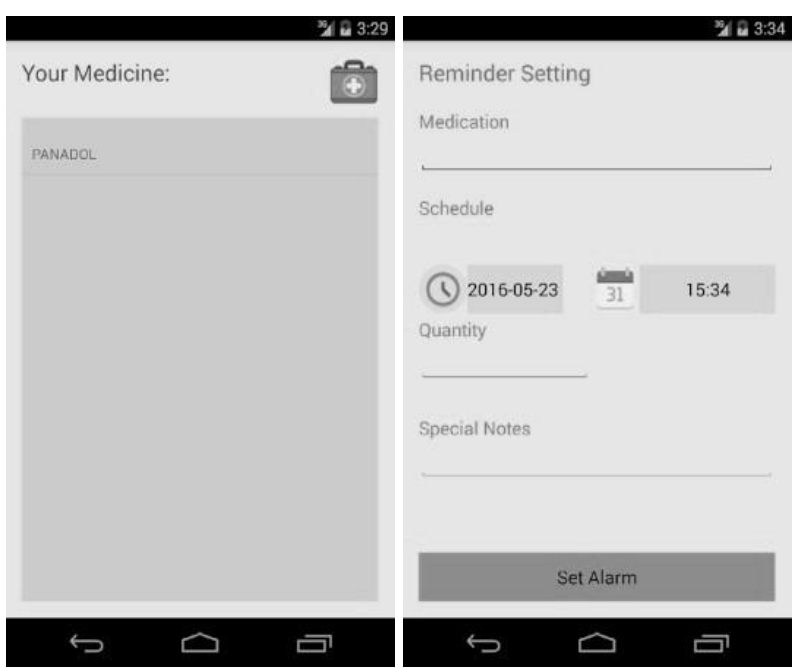

Fig. 7. (a) Reminder list. (b) medicine intake reminder setup (Patient).

After a reminder have been set, a notification will pop-up to remind patients for medicine intake. Figure 8 shows the notification reminder for medicine intake.

\section{Medicine Intake Reminder}

It is time for your medicine intake.

Fig. 8. Notification reminder for medicine intake (Patient).
Pseudocode 1 represents the workflow for medication intake reminder set by the patients. Pseudocode 2 represents the workflow for appointment reminder with the physician set by the clinician.
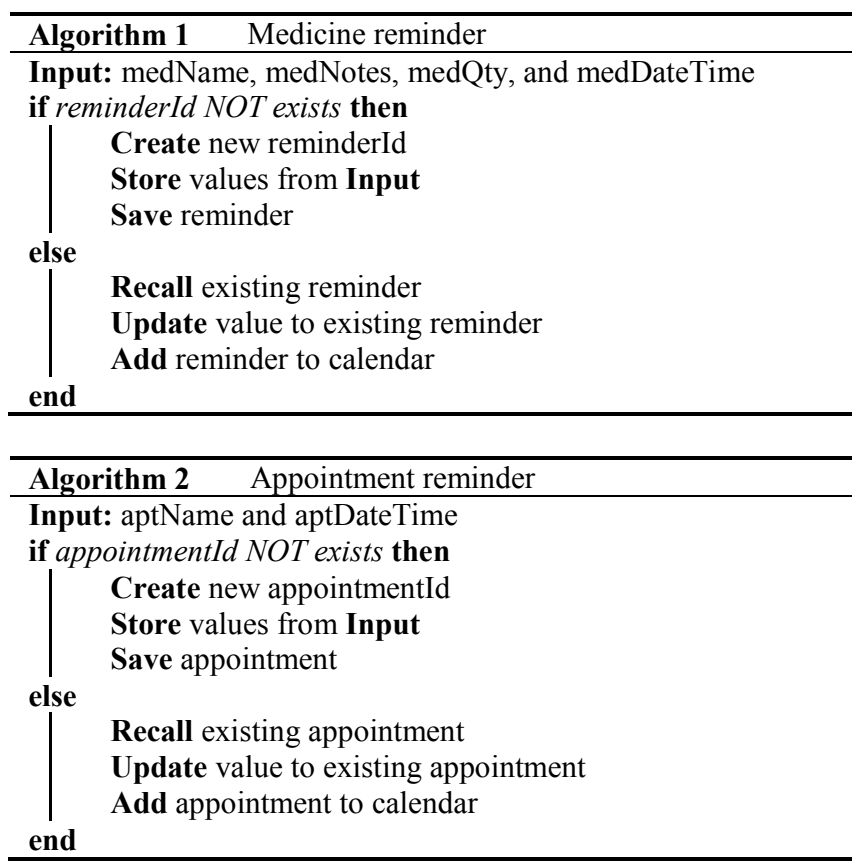

The above pseudocodes are vital to the success of this application where empowering self-management initiatives and medical intervention.

\section{Result and Discussion}

The developed application was deployed and installed into an Android system phones for system testing. The tests ensure the functionality of the modules perform properly and error-free. These tests involve the users; administrator and patients, covering all modules available according to the user access level.

After the system functionality testing is completed, users are invited to participate in user acceptance testing (UAT) to get feedback on whether the system and applications meet the user requirement. UAT is considered as the last phase of any software development process that helps in making sure that the software or applications can handle its required tasks in real world. UAT has provided users with the chance to interact with MedsBox Reminder and provide feedback to the development team for enhancement and bug-fixes before the final release of application.

UAT was conducted by selecting patients that are accustomed to the service received from a clinic. The questionnaire form was distributed to the respondents while testing the prototype. The UAT form consists of three sections: tester's demographic, application UI/UX UAT, and application functionality UAT. Table 1 shows the questions and criteria for scoring the UAT. 
Table 1. UAT questions and criteria of scoring.

\begin{tabular}{|l|c|c|}
\hline Section 1: Tester's Demographic & Male & Female \\
\hline Gender & $84 \%$ & $16 \%$ \\
\hline Age (in years) & $18-55$ & $24-36$ \\
\hline Section 2: Interface and Experience & Agree & Neutral \\
\hline Overall application design & $76 \%$ & $24 \%$ \\
\hline Suitable background and colour & $92 \%$ & $8 \%$ \\
\hline Suitable font family and font size & $78 \%$ & $22 \%$ \\
\hline Contents are clear and readable & $90 \%$ & $10 \%$ \\
\hline Smooth system flow and easily used & $78 \%$ & $22 \%$ \\
\hline All buttons and links are working & $88 \%$ & $22 \%$ \\
\hline Section 3: Application Functionality & Agree & Neutral \\
\hline Suitable for monitoring medicine intake & $60 \%$ & $40 \%$ \\
\hline Reminder notification works well & $60 \%$ & $40 \%$ \\
\hline Overall application functionality & $80 \%$ & $20 \%$ \\
\hline
\end{tabular}

The implementation of system testing is successfully conducted with the clinicians and the obtained results is compared to the expected results. Comments and suggestions were gathered for future enhancement. Table 2 summarizes the UAT result from respondents.

Table 2. Overall UAT results from respondents.

\begin{tabular}{|l|c|c|c|}
\hline Criterion of Score & Agree & Neutral & Disagree \\
\hline Intuitive user interface & $82 \%$ & $18 \%$ & $0 \%$ \\
\hline $\begin{array}{l}\text { Functions well as } \\
\text { intended }\end{array}$ & $67 \%$ & $33 \%$ & $0 \%$ \\
\hline \begin{tabular}{l} 
Overall score (average) \\
\hline
\end{tabular} & $78 \%$ & $22 \%$ & $0 \%$ \\
\hline
\end{tabular}

Overall, MedsBox Reminder application has achieved the objective to be a well-functioning application which helps user in managing their daily medication intake. However, there is still room for improvements in making MedsBox Reminder better in the future.

\section{Conclusion}

The enhancement of mobile technologies initiates opportunities in the M-Health field especially for selfmanagement of individual health, immediate medical interventions and assistance from medical practitioners, and also health awareness at an individual level. MedsBox Reminder application was developed to prove that M-Health is not only practical for improvement of the overall quality of life, but also much feasible for development and brings benefits to both health care industries and the community.

This work is supported by the Fundamental Research Grant (Vot 1612) from Ministry of Higher Education Malaysia, Universiti Tun Hussein Onn Malaysia (UTHM), and GATES IT Solution Sdn. Bhd.

\section{References}

1. World Health Organization Media Centre, Diabetes, http://www.who.int/mediacentre/factsheets/fs312/en /. Retrieved on October 27, 2016 (2015)

2. L. Whitehead, P. Seaton, J. Med. Internet Res. 18, 5 (2016)

3. V. Zoffmann, A. Hörnsten, S. Storbækken, M. Graue, B. Rasmussen, A. Wahl, M. Kirkevold, Patient Educ. Couns. 99, 400-407 (2016)

4. R.S. Istepanian, E. Jovanov, Y.T. Zhang, IEEE Trans. Inf, Technol. Biomed. 8, 405-414 (2004)

5. WHO Global Observatory for eHealth. New horizons for health through mobile technologies. Geneva: World Health Organization; 2011:112.

6. Govette, J. 30 Amazing Mobile Health Technology Statistics for Today's Physician, https:/getreferralmd.com/2015/08/mobile-

healthcare-technology-statistics/. Retrieved on October 27, 2016 (2015)

7. C.E. Smith, U. Piamjariyakul, M. Werkowitch, D.M. Yadrich, N. Thompson, D. Hooper, E.L. Nelson, Int. J. Sens. Netw. Data Commun. 5, 1 (2016)

8. A.V. Heerden, M. Tomlinson, L. Swartz, Bull. World Health Organ. 90, 393-394 (2012)

9. A. Rehalia, S. Prasad, mHealth 2, 3 (2016)

10. K. Lorig, P.L. Ritter, R.M. Turner, K. English, D.D. Laurent, J. Greenberg, J. Med. Internet Res. 18, 6 (2016)

11. C. Jenkins, N.S. Burkett, B. Ovbiagele, M. Mueller, S. Patel, B. Brunner-Jackson, R. Saulson, F. Treiber, mHealth 2, 5 (2016)

12. A.L. Hartzler, A. Venkatakrishnan, S. Mohan, M. Silva, P. Lozano, J.D. Ralston, E. Ludman, D. Rosenberg, K.M. Newton, L. Nelson, P. Pirolli, IEEE Trans. Inf. Technol. Biomed. 2016, 3277-3281 (2016) 
13. Z.R. Nandi, A.C. Olivera, R.E.C. Valencia, A.A. Paredes, G.A. Alonso, Panam. Healt. Care 2015, 1-4 (2015)

14. A.D. Nguyen, M.T. Baysari, D.R. Kannangara, A. Tariq, A.Y. Lau, J.I. Westbrook, R.O. Day, Int. J. Med. Inform. 94, 67-74 (2016)

15. I. de la Torre-Díez, B. Martínez-Pérez, M. LópezCoronado, J.J. Rodrigues, J. Arambarri, Iber. Conf. Inf. Syst. 2016, 1-5 (2016)

16. J.L. Peck, M. Stanton, G.E. Reynolds, J. Pediatr. Health Car. 28, 35.42 (2014)

17. O. Oluwagbemi,F. Oluwagbemi, C. Ughamadu, Info. Med. Unlocked 2, 38-69 (2016)

18. P. Parmar, R. Krishnamurthi, M.A. Ikram, A. Hofman, S.S. Mirza, Y. Varakin, M. Kravchenko, M. Piradov, A.G. Thrift, B. Norrving, W. Wang, Int. J. Stroke 10, 231-244 (2015)
19. P. Divall, J. Camosso-Stefinovic, R. Baker, Health Inform. J. 19, 16-28 (2013)

20. M. Othman, N. Kasabov, E. Tu, V. Feigin, R. Krishnamurthi, Z. Hou, Y. Chen, J. Hu, IEEE IJCNN 2014, 3197-3204 (2014)

21. N. Kasabov, N.M. Scott, E. Tu, S. Marks, N. Sengupta, E. Capecci, M. Othman, M.G. Doborjeh, N. Murli, R. Hartono, J.I. Espinosa-Ramos, L. Zhou, F.B. Alvi, G. Wang, D. Taylor, V. Feigin, S. Gulyaev, M. Mahmoud, Z.G. Hou, J. Yang, Neural Networks 78, 1-14 (2016)

22. S.V. Zanjal, G.R. Talmale, Procedia Compt. Sci. 78, 471-476 (2016)

23. K. Kauppi, K.A. Kannisto, H. Hätönen, M. Anttila, E. Löyttyniemi, C.E. Adams, M. Välimäki, Schizophr. Res. 168, 514-522 (2015) 